\title{
Superspace formulations of the (super)twistor string
}

\author{
Igor A. Bandos ${ }^{\dagger *}$, José A. de Azcárraga ${ }^{\dagger}$ and Cèsar Miquel-Espanya ${ }^{\dagger}$ \\ ${ }^{\dagger}$ Departamento de Física Teórica, Univ. de Valencia and IFIC (CSIC-UVEG), 46100-Burjassot (Valencia), Spain \\ *Institute for Theoretical Physics, NSC "Kharkov Institute of Physics and Technology", UA61108, Kharkov, Ukraine
}

\begin{abstract}
The superspace formulation of the worldvolume action of twistor string models is considered. It is shown that for the Berkovits-Siegel closed twistor string such a formulation is provided by a $\mathrm{N}=4$ twistor-like action of the tensionless superstring. A similar inverse twistor transform of the open twistor string model (Berkovits model) results in a dynamical system containing two copies of the $D=4, N=4$ superspace coordinate functions, one left-moving and one right-moving, that are glued by the boundary conditions.

We also discuss possible candidates for a tensionful superstring action leading to the twistor string in the tensionless limit as well as multidimensional counterparts of twistor strings in the framework of both 'standard' superspace and superspace enlarged by tensorial coordinates (tensorial superspaces), which constitute a natural framework for massless higher spin theories.
\end{abstract}

PACS numbers: 11.30.Pb, 11.25.-w, 11.10.Kk, 12.60Jv;

FTUV-06-0405 , IFIC/06-06 ; April 5, 2006

\section{INTRODUCTION}

The connection between Yang-Mills and string theories was reconsidered in 1] using the twistor approach [2] (see [3] for an earlier study). It was originally noticed that a class of perturbative tree amplitudes for the gauge fields of $N=4$ supersymmetric Yang-Mills theories were reproduced from a string moving in the projective $\mathbb{C P}^{(3 \mid 4)}$ superspace. $\mathbb{C P}^{(3 \mid 4)}$ is a Calabi-Yau supermanifold, the bosonic body of which, $\mathbb{C P}^{3}$, is Penrose twistor space 2].

The class of Yang-Mills amplitudes that may be described by the twistor string model 1] was then extended in [4, 5]. In particular, one loop amplitudes are also amenable to a twistor presentation using a technique [5, 6, 7] suggested by the twistor string approach. This further supports the original idea of the existence of a deep connection between the supertwistor string and $N=4$ super-Yang-Mills gauge theories in the usual $D=4$ (super)spacetime. The restrictions on a possible full identification of these models come from the impossibility of isolating the closed string sector of the twistor string from the open one, and from the observation that the closed string sector, in view of its conformal invariance, should lead to conformal supergravity [8], which has itself problems for its physical treatment. Recently [9], a twistor string-like generating functional for superYang-Mills amplitudes was derived from a twistor reformulation of the super-Yang-Mills action which, in turn, followed from its 'asymmetric' formulation [10]. An analogous formulation for gravity that might be related with the closed twistor string was considered in 11].

At the same time, alternative twistor string models were proposed in [12, 13] and 14]. It was argued in [13] that the twistor string might be related with the $\mathcal{N}=2$ spinning string, which has $\mathcal{N}=2$ extended worldvolume supersymmetry and is defined in a $D=4$ spacetime with two time-like directions (see [15] for further discussion). The problem of relating the twistor string with two-time physics was considered in [16] where possible higher di- mensional generalizations were also discussed.

To look for a (super)spacetime formulation of the twistor string that originally had been given only in terms of supertwistor variables but not in the usual spacetime or superspace coordinates, Siegel proposed a new twistor string action [14] in terms of the Atiyah-Drinfel'dHitchin-Manin (ADHM) [1] (super)twistors (see [18]).

One of the messages of this paper is that the standard (not ADHM) twistor superstring action, at least the closed string chiral version of Siegel [14], can be rewritten in terms of superspace coordinates in a different manner using a method similar to the change of variables that relates the different forms of the Ferber-Shirafuji superparticle action [19] (see also 20]). The spacetime/superspace action that is classically equivalent to the twistor superstring [14] has $8 \kappa$-symmetries and turns out to be a straightforward $N=4$ generalization of the $D=4$ tensionless superstring action in [21, 22].

A similar twistor transform of the Berkovits model for the open twistor string results in an action formulated in terms of two copies of the coordinate functions of $D=4$, $N=4$ superspace. We notice in passing that such a set of variables, albeit for $N=1$, was used in [23] to write an equivalent form of the $N=2$ Green-Schwarz superstring action.

The way of quantizing the twistor string discussed in [1, 8, 12, 14] makes it clear that this string should be understood as the tensionless limit of a tensionful string model. The reason is that in the case of the intrinsically tensionless superstring ('null-superstring') its conformal invariance is maintained by a continuous mass spectrum [21, 24], while the tensionless limit of a tensionful string rather contains a set of massless fields [25, 26, 27]. The relation of such a tensionful model with the so-called QCD string 28] was discussed in 14], but in a purely bosonic context. The correspondence with the nullsuperstring of [21, 22] makes transparent that the $N=1$ $(N=2)$ counterparts of the twistor string action appear in the tensionless limit of the standard $N=1(N=2)$ 
Green-Schwarz superstring models in $D=4$. The search for a tensionful parent action for the standard (superYang-Mills related) $N=4$ twistor string will lead us naturally to enlarge the $D=4, N=4 \Sigma^{(4 \mid 4 N)}$ superspace to the $D=10, N=1$ superspace $\Sigma^{(10 \mid 16)}$ or to a tensorial superspace $\widetilde{\Sigma}^{(4+6 \mid 16)}$ with additional antisymmetric tensor coordinates.

\section{SUPERTWISTOR STRING MODELS}

\section{A. Siegel's closed string action}

To our knowledge, there are at present four versions of the supertwistor string action, that of Witten [1], a constrained sigma model the tangent superspace of which is $\mathbb{C P}{ }^{(3 \mid 4)}$, the one put forward by Berkovits 12$]$ involving two supertwistors, and the two proposed by Siegel in [14].

The simplest action is that of the closed twistor string model of the first part of ref. 14] (we do not discuss here the second, ADHM twistor action given in [14], which includes explicitly the spacetime coordinates and that in this sense 'untwists' the twistor superstring). It is given by

$$
\begin{aligned}
S & =\int_{W^{2}} e^{++} \wedge \bar{\Upsilon}_{\Sigma} \nabla \Upsilon^{\Sigma}+d^{2} \xi L_{G}= \\
& =\int d^{2} \xi\left[\sqrt{|\gamma(\xi)|} \bar{\Upsilon}_{\Sigma}(\xi) \nabla_{--} \Upsilon^{\Sigma}(\xi)+L_{G}\right]
\end{aligned}
$$

where $e^{ \pm \pm}=d \xi^{m} e_{m}^{ \pm \pm}(\xi)$ are the worldsheet zweibein one-forms and $e^{++} \wedge e^{--}=d^{2} \xi \sqrt{|\gamma|}$ is the invariant surface element of the worldsheet $W^{2}$. The basic worldsheet fields

$$
\begin{array}{r}
\Upsilon^{\Sigma}=\left(\mu^{\dot{\alpha}}, \lambda_{\alpha} ; \eta_{i}\right)=\Upsilon^{\Sigma}(\xi), \\
\alpha=1,2, \quad \dot{\alpha}=1,2, \quad i=1,2,3,4,
\end{array}
$$

determine the $(N=4)$ Ferber supertwistor [19] $\Upsilon^{\Sigma}$ and

$$
\begin{aligned}
& \bar{\Upsilon}_{\Sigma}:=\left(\Upsilon^{\Pi}\right)^{*} \Omega_{\Pi \Sigma}=\left(\bar{\lambda}_{\dot{\alpha}},-\bar{\mu}^{\alpha} ; 2 i \bar{\eta}^{i}\right), \\
& \alpha=1,2, \quad \dot{\alpha}=1,2, \quad i=1,2,3,4
\end{aligned}
$$

62] is defined through the $S U(2,2 \mid 4)$-invariant tensor

$$
\Omega_{\Sigma \Pi}=\left(\begin{array}{ccc}
0 & -\delta_{\dot{\alpha}}^{\dot{\beta}} & 0 \\
\delta^{\alpha}{ }_{\beta} & 0 & 0 \\
0 & 0 & 2 i
\end{array}\right)
$$

Finally,

$$
\nabla=e^{++} \nabla_{++}+e^{--} \nabla_{--}=d-i B
$$

is the worldsheet covariant derivative with the $U(1)$ connection $B$. In (2.1), $L_{G}$ is the action for the worldsheet fields that are used to construct the Yang-Mills symmetry current (one can use e.g., the worldsheet fermionic degrees of freedom, as discussed below).
The target supermanifold $\mathbb{C P}^{(3 \mid 4)}$ of the $N=4$ supertwistors (2.2), which generalize the Penrose twistors [2], defines a fundamental representation of the $S U(2,2 \mid 4)$ superconformal group $(S U(2,2) \sim S O(2,4))$. Thus, the action (3.1) is superconformally $(S U(2,2 \mid 4)$-) invariant by construction. Such an action can be also written for $N \neq 4$ supertwistors; in this case it possesses $S U(2,2 \mid N)$ superconformal symmetry, but $\mathbb{C P}^{(d \mid N)}$ is a Calabi-Yau manifold only if $d+1=N$. The set of special properties of the $N=4$ case includes the existence of the holomorphic integral measure on $\mathbb{C P}^{(3 \mid 4)}$,

$$
\begin{gathered}
\Omega_{(3 \mid 4)}=\Omega_{(3 \mid 0)} \epsilon_{i j k l} \frac{\partial}{\partial \eta_{i}} \frac{\partial}{\partial \eta_{j}} \frac{\partial}{\partial \eta_{k}} \frac{\partial}{\partial \eta_{l}}, \\
\Omega_{(3 \mid 0)}=\epsilon_{\alpha^{\prime} \beta^{\prime} \gamma^{\prime} \delta^{\prime}} \Upsilon^{\alpha^{\prime}} d \Upsilon^{\beta^{\prime}} \wedge d \Upsilon^{\gamma^{\prime}} \wedge d \Upsilon^{\delta^{\prime}},
\end{gathered}
$$

where $\alpha^{\prime}=1, \ldots, 4(=1,2, \dot{1}, \dot{2})$. The $\Omega_{(3 \mid 4)}$ integral form is invariant under the $U(1)$-phase transformations of the twistors,

$$
\Upsilon^{\Sigma} \mapsto e^{i \beta} \Upsilon^{\Sigma}, \quad \bar{\Upsilon}_{\Sigma} \mapsto e^{-i \beta} \bar{\Upsilon}_{\Sigma}
$$

and also under the scaling

$$
\Upsilon^{\Sigma} \mapsto e^{\beta^{\prime}} \Upsilon^{\Sigma} .
$$

These two transformations are also symmetries of the action (2.1) provided the scaling of the twistors, Eq. (2.8), is supplemented by the scaling of the vielbein form

$$
e^{++} \mapsto e^{-2 \beta^{\prime}} e^{++} .
$$

Because of the covariant derivative 2.5), the $U(1)$ gauge transformations (2.7) (now with local parameter $\beta(\xi)$ under which $B \mapsto B+d \beta)$ are a gauge symmetry of the action (2.1). On the other hand, the rôle of this $U(1)$ connection $B$ is analogous (as noted in [14]) to that of the auxiliary worldsheet metric in the standard superstring model. Namely, its equations of motion impose on the supertwistor $\Upsilon$ the constraint

$$
\bar{\Upsilon}_{\Sigma} \Upsilon^{\Sigma}=\bar{\lambda}_{\dot{\alpha}} \mu^{\dot{\alpha}}-\bar{\mu}^{\alpha} \lambda_{\alpha}+2 i \bar{\eta}^{i} \eta_{i}=0
$$

which, in the Hamiltonian framework, is the generator of the $U(1)$ symmetry (2.7).

As the constraint (2.10) appears as a non-dynamical equation of motion for the auxiliary field $B$, one can consider the action

$$
\begin{aligned}
S & =\int e^{++} \wedge \bar{\Upsilon}_{\Sigma} d \Upsilon^{\Sigma}+d^{2} \xi L_{G}= \\
& =\int e^{++} \wedge\left(\bar{\lambda}_{\dot{\alpha}} d \mu^{\dot{\alpha}}-\bar{\mu}^{\alpha} d \lambda_{\alpha}+2 i \bar{\eta}^{i} d \eta_{i}\right)+d^{2} \xi L_{G},
\end{aligned}
$$

where the supertwistor variables are constrained by (2.10), as an alternative to (2.1). In this form the action does not contain the connection $B$, but the $U(1)$ gauge symmetry still holds due to the constraint (2.10). This constraint also makes the action (2.11) invariant under the local worldsheet Lorentz $S O(1,1)$, which here is equivalent to the scaling or local $G L(1, \mathbb{R}))$ symmetry (2.8) 63]. 


\section{B. On the Yang-Mills current part of the action}

The simplest choice for $L_{G}$ in (2.1) is the free fermion action which allows one to construct the current $\mathcal{J}^{r}=$ $\bar{\psi}_{J} T_{I}^{r J} \psi^{I}$ for the Yang-Mills gauge group $G$ (where $T_{I}^{r}$ is the matrix representation for its infinitesimal generators) and, hence, to describe the coupling of the string to the Yang-Mills gauge field, $\int \operatorname{tr} \mathcal{J} \mathcal{A} \propto \int \mathcal{J}^{r} \mathcal{A}^{r}$, according to 29, 30, 31]. In the action (2.1) for the closed string [14], these free fermions should have the same twodimensional (worldsheet) 'chirality' as the supertwistor variables 32 . Thus the Lagrangian including the vertex operator reads

$$
d^{2} \xi\left[L_{G}+\operatorname{tr}(\mathcal{J} \mathcal{A})\right]=\frac{1}{2} e^{++} \wedge\left(\bar{\psi}_{I} D \psi^{I}-D \bar{\psi}_{I} \psi^{I}\right),
$$

where $D$ is the Yang-Mills covariant derivative $D \psi^{I}=$ $d \psi^{I}+\mathcal{A}^{I}{ }_{J} \psi^{J}$ and $\bar{\psi}_{I}=\left(\psi^{J}\right)^{*} \mathcal{C}_{J^{*} I}$ with $\mathcal{C}_{J^{*} I}$ being invariant under the gauge group $G$; for instance $\mathcal{C}_{J^{*} I}=\delta_{J^{*} I}$ for $G=U(m)$.

\section{Berkovits's open string action}

Berkovits's open string version of the twistor string [12] contains two supertwistor fields, a left moving $\Upsilon^{-\Sigma}$ and a right moving $\Upsilon^{+\Sigma}$,

$$
\begin{aligned}
& \Upsilon^{-\Sigma}=\left(\mu^{-\dot{\alpha}}, \lambda_{\alpha}^{-} ; \eta_{i}^{-}\right), \\
& \Upsilon^{+\Sigma}=\left(\mu^{+\dot{\alpha}}, \lambda_{\alpha}^{+} ; \eta_{i}^{+}\right) .
\end{aligned}
$$

The worldsheet action reads

$$
\begin{aligned}
S= & \int_{W^{2}} e^{++} \wedge \bar{\Upsilon}_{\Sigma}^{-} \nabla\left(\Upsilon^{-\Sigma}\right)-e^{--} \wedge \bar{\Upsilon}_{\Sigma}^{+} \nabla\left(\Upsilon^{+\Sigma}\right)+ \\
& +\int_{W^{2}} d^{2} \xi L_{G}
\end{aligned}
$$

where $L_{G}$ is the Lagrangian for the YM current (e.g. $\frac{1}{2} e^{++} \wedge\left(\bar{\psi}_{+I} d \psi_{+}^{I}-d \bar{\psi}_{+I} \psi_{+}^{I}\right)+\frac{1}{2} e^{--} \wedge\left(\bar{\psi}_{-I} d \psi_{-}^{I}-\right.$ $\left.d \bar{\psi}_{-I} \psi_{-}^{I}\right)$ for the left and right fermionic fields $\left.\psi_{-}, \psi_{+}\right)$. The above notation explains why the worldsheet supervielbein forms were denoted by $e^{++}$and $e^{--}$: the double sign superscript indicates $S O(1,1)$-vector transformation properties $\left(e^{ \pm \pm} \rightarrow e^{\mp 2 \beta^{\prime}} e^{ \pm \pm}\right)$, while the single \pm superscripts were reserved for supertwistors $\Upsilon^{ \pm \Sigma}$ to indicate their spinor $\left(\Upsilon^{ \pm} \rightarrow e^{\mp \beta^{\prime}} \Upsilon^{ \pm}\right)$transformation properties under the worldsheet Lorentz group.

The action (2.14) assumes boundary conditions that identify, in particular, the left and the right supertwistors 64] on the worldsurface boundary $\partial W^{2}$ :

$\left.\Upsilon^{-\Sigma}\right|_{\partial W^{2}}=\left.\Upsilon^{+\Sigma}\right|_{\partial W^{2}},\left.\quad \bar{\Upsilon}_{\Sigma}^{-}\right|_{\partial W^{2}}=\left.\bar{\Upsilon}_{\Sigma}^{+}\right|_{\partial W^{2}}$,

as well as the left and right currents. Siegel [14] motivated his modification (2.1) of the Berkovits twistor string (2.14) by stating that the boundary conditions do not play any rôle except that of halving the number of the twistor degrees of freedom. Specifically, the identification (2.15) of the two supertwistors on the boundary $\partial W^{2}$ of $W^{2}$ allows one to construct all the open string vertex operators using only one set of twistor variables, either $\left(\Upsilon^{-\Sigma}, \bar{\Upsilon}_{\Sigma}^{-}\right)$or $\left(\Upsilon^{+\Sigma}, \bar{\Upsilon}_{\Sigma}^{+}\right)$. It was also noticed that the closed string version is more natural for a spacetime interpretation, which was constructed by moving from the Penrose-twistor string to an alternative, 'six dimensional' ADHM-twistor string [14]. We will present here another, more straightforward way to arrive at the spacetime or, more precisely, at the standard superspace presentation.

\section{Witten's action}

For completeness we describe here the original Witten's proposal [1] for a $\mathbb{C P}^{(3 \mid 4)}$ twistor string. It uses only one supertwistor and it is based on the following action

$$
\begin{aligned}
S_{W} & =\int_{W^{2}}\left[\nabla \bar{\Upsilon}_{\Sigma} \wedge * \nabla \Upsilon^{\Sigma}+d^{2} \xi \Xi(\xi)\left(\bar{\Upsilon}_{\Sigma} \Upsilon^{\Sigma}-r\right)\right]= \\
= & \int e^{++} \wedge e^{--}\left[\nabla_{++} \bar{\Upsilon}_{\Sigma} \nabla_{--} \Upsilon^{\Sigma}+\nabla_{--} \bar{\Upsilon}_{\Sigma} \nabla_{++} \Upsilon^{\Sigma}\right] \\
& +\int d^{2} \xi \Xi(\xi)\left(\bar{\Upsilon}_{\Sigma} \Upsilon^{\Sigma}-r\right)
\end{aligned}
$$

describing a $\mathbb{C P}^{(3 \mid 4)}$ sigma-model subject to the additional constraint

$$
\bar{\Upsilon}_{\Sigma} \Upsilon^{\Sigma}=r
$$

for some constant $r$, introduced into the action through the Lagrange multiplier $\Xi(\xi)$. In Eq. (2.16), * is the Hodge operator for the auxiliary worldsheet metric,

$$
* e^{--}=e^{--}, \quad * e^{++}=-e^{++} .
$$

In the case of particle mechanics [19] the modification of the twistor constraint (2.10) by a nonvanishing $r$ (Eq. (2.17) is known to describe a massless particle with helicity $s=r / 2$. On the other hand, it is also known that, due to the noncommutativity of $\Upsilon^{\Sigma}$ and $\bar{\Upsilon}_{\Sigma}$ in a quantum description, the classical constraint (2.10) can also lead after quantization to (2.17) with a nonvanishing $r$ 65].

The covariant derivative $\nabla$ in (2.16) contains the U(1)gauge field $B: \quad \nabla \Upsilon_{\Sigma}=d \Upsilon_{\Sigma}-i B \Upsilon_{\Sigma}$, Eq. (2.5). Due to the constraint (2.17), the equations of motion for the gauge field $B$ can be written as

$$
\begin{aligned}
0=\bar{\Upsilon}_{\Sigma} \nabla \Upsilon^{\Sigma} & =\bar{\Upsilon}_{\Sigma} d \Upsilon^{\Sigma}-i B \bar{\Upsilon}_{\Sigma} \Upsilon^{\Sigma}= \\
& =d \Upsilon^{\Sigma} \bar{\Upsilon}_{\Sigma}-i B r .
\end{aligned}
$$

Hence for a nonvanishing $r$ the $\mathrm{U}(1)$-gauge field may be expressed in terms of the supertwistor and its conjugate,

$$
B=-\frac{i}{r} \bar{\Upsilon}_{\Sigma} d \Upsilon^{\Sigma}
$$


In this case the Lagrange multiplier $\Xi(\xi)$ is also expressed in terms of the twistor by the solution

$$
d^{2} \xi \Xi=+\frac{1}{r} \nabla \bar{\Upsilon}_{\Sigma} \wedge * \nabla \Upsilon^{\Sigma}
$$

of the supertwistors equations of motion

$$
\begin{aligned}
& \nabla * \nabla \Upsilon^{\Sigma}=d^{2} \xi \Xi \Upsilon^{\Sigma} \\
& \nabla * \nabla \bar{\Upsilon}_{\Sigma}=d^{2} \xi \Xi \bar{\Upsilon}_{\Sigma}
\end{aligned}
$$

[To arrive at (2.21) one uses the constraint (2.17) and equations (2.19)]. Notice that inserting (2.21) back into the equations of motion (2.22) one finds

$$
\nabla * \nabla \Upsilon^{\Sigma}=-\frac{1}{r} \Upsilon^{\Sigma} \nabla \bar{\Upsilon}_{\Pi} \wedge * \nabla \Upsilon^{\Pi}
$$

and its c.c. expression. The r.h.s. is proportional to the trace of the energy-momentum tensor $T_{m n} \propto$ $\nabla_{m} \bar{\Upsilon}_{\Pi} \nabla_{n} \Upsilon^{\Pi}$. This vanishes if the equation of motion for the auxiliary worldvolume metric $\gamma_{m n}=e_{(m}^{++} e_{n)}^{--}$is taken into account.

If $r=0$, Eq. (2.20) does no longer follow from (2.19) as $B$ does not appear in (2.19). Then, although the Lagrange multiplier $\Xi$ is still present in the dynamical equations (2.22), their contractions with $\bar{\Upsilon}_{\Sigma}$ and $\Upsilon^{\Sigma}$ cannot be used to express $\Xi$ in terms of the covariant derivatives of supertwistors, like in (2.21), but it rather produces

$$
\bar{\Upsilon}_{\Sigma} \nabla * \nabla \Upsilon^{\Sigma}=0, \quad \nabla * \nabla \bar{\Upsilon}_{\Sigma} \Upsilon^{\Sigma}=0
$$

In the light of Eq. (2.19), Eqs. (2.24) imply

$$
\nabla \bar{\Upsilon}_{\Sigma} \wedge * \nabla \Upsilon^{\Sigma}=0 \text { for } r=0
$$

The existence of the invariant integral form 2.6 makes the $N=4$ supertwistor space $\mathbb{C P}^{(3 \mid 4)}$ a Calabi-Yau supermanifold; this is needed to relate the sigma model to the topological 'B-model' 1]. To reproduce the MHV amplitudes of the Yang-Mills theory the twistor string model based on Eq. (2.16) has to be enriched by Dinstanton contributions [1] (that the Berkovits model seeks to avoid). We will not need nor consider these details below.

\section{III. $D=4 N=4$ SUPERSPACE FORMULATION OF THE SUPERTWISTOR STRING}

\section{A. Siegel's closed supertwistor string model as a model in $\mathrm{D}=4 \mathrm{~N}=4$ superspace}

We show here that the (non Yang-Mills part of) Siegel's closed twistor string, $e^{++} \wedge \bar{\Upsilon}_{\Sigma} \nabla \Upsilon^{\Sigma}$ in Eq. (2.1), has a transparent $D=4, N=4$ superspace form,

$$
S_{S}=\int_{W^{2}}\left[e^{++} \wedge \hat{\Pi}^{\dot{\alpha} \alpha} \bar{\lambda}_{\dot{\alpha}} \lambda_{\alpha}+d^{2} \xi L_{G}\right],
$$

where $\hat{\Pi}^{\dot{\alpha} \alpha} \equiv d \xi^{m} \Pi_{m}^{\dot{\alpha} \alpha} \equiv d \tau \Pi_{\tau}^{\dot{\alpha} \alpha}+d \sigma \Pi_{\sigma}^{\dot{\alpha} \alpha}$ is the pull-back to the worldsheet $W^{2}$ of the flat supervielbein on $D=4$, $N=4$ superspace,

$$
\Pi^{\dot{\alpha} \alpha}:=d x^{\dot{\alpha} \alpha}-i d \theta_{i}^{\alpha} \bar{\theta}^{\dot{\alpha} i}+i \theta_{i}^{\alpha} d \bar{\theta}^{\dot{\alpha} i},
$$

where $i=1,2,3,4 . \Pi^{\dot{\alpha} \alpha}$ can also be written in terms of a left or right chiral coordinate basis,

$$
\begin{aligned}
\Pi^{\dot{\alpha} \alpha}:= & d x_{L}^{\dot{\alpha} \alpha}-2 i d \theta_{i}^{\alpha} \bar{\theta}^{\dot{\alpha} i}=d x_{R}^{\alpha \dot{\alpha}}+2 i \theta_{i}^{\alpha} d \bar{\theta}^{\dot{\alpha} i}, \\
& x_{L}^{\dot{\alpha} \alpha}:=x^{\dot{\alpha} \alpha}+i \theta_{i}^{\alpha} \bar{\theta}^{\dot{\alpha} i}=\left(x_{R}^{\dot{\alpha} \alpha}\right)^{*}, \\
& x_{R}^{\dot{\alpha} \alpha}:=x^{\dot{\alpha} \alpha}-i \theta_{i}^{\alpha} \bar{\theta}^{\dot{\alpha} i}=\left(x_{L}^{\dot{\alpha} \alpha}\right)^{*} .
\end{aligned}
$$

The bosonic spinors $\lambda_{\alpha}, \bar{\lambda}_{\dot{\alpha}}$ in (3.1) are auxiliary. Their equations of motion

$$
e^{++} \wedge \Pi^{\dot{\alpha} \alpha} \lambda_{\alpha}=0 \quad \text { or } \quad \Pi_{--}^{\dot{\alpha} \alpha} \lambda_{\alpha}=0
$$

are non-dynamical and imply

$$
\Pi_{--}^{\dot{\alpha} \alpha} \sim \bar{\lambda}^{\dot{\alpha}} \lambda^{\alpha}
$$

which solves the Virasoro constraint

$$
\Pi_{--}^{\dot{\alpha} \alpha} \Pi_{--\dot{\alpha} \alpha}=0 \text {. }
$$

It may be easily checked that the action 3.1 is equivalent to (2.1). Indeed, by using Leibniz rule $\left(d x^{\dot{\alpha} \alpha} \lambda_{\alpha}=\right.$ $d\left(x^{\dot{\alpha} \alpha} \lambda_{\alpha}\right)-x^{\dot{\alpha} \alpha} d \lambda_{\alpha}$, etc.) Eq. (3.1) can be written in the form

$$
\begin{aligned}
S & =\int e^{++} \wedge\left(d \mu^{\dot{\alpha}} \bar{\lambda}_{\dot{\alpha}}-d \lambda_{\alpha} \bar{\mu}^{\alpha}-2 i d \eta_{i} \bar{\eta}^{i}\right)+d^{2} \xi L_{G} \\
& =\int e^{++} \wedge d\left(\Upsilon^{\Sigma}\right) \bar{\Upsilon}_{\Sigma}+d^{2} \xi L_{G},
\end{aligned}
$$

where the components of the supertwistor are related to the superspace coordinates by the following supersymmetric generalization [19] of the Penrose incidence relation 2]

$$
\mu^{\dot{\alpha}}=x_{L}^{\dot{\alpha} \alpha} \lambda_{\alpha}:=\left(x^{\dot{\alpha} \alpha}+i \theta_{i}^{\alpha} \bar{\theta}^{\dot{\alpha} i}\right) \lambda_{\alpha}, \quad \eta_{i}=\theta_{i}^{\alpha} \lambda_{\alpha} .
$$

Eqs. (3.10) give the general solution of the constraint $\bar{\Upsilon}_{\Sigma} \Upsilon^{\Sigma}=0$, which allows us to use Eq. (2.10) instead of (3.10).

\section{B. $\kappa$-symmetry}

The action (3.1), involving the superspace coordinate fields and the auxiliary bosonic spinor fields $\lambda(\xi), \bar{\lambda}(\xi)$, is thus equivalent to the twistor action (3.9). Ignoring the Yang-Mills current variables in $L_{G}$ and the auxiliary one-form $e^{++}$, one sees that the superspace action (3.1) contains, besides the auxiliary $e^{++}, \mathbf{8}(4+4)$ real bosonic and $\mathbf{1 6}(4 \times 4)$ real fermionic variables, while the twistor one (3.9) contains instead 8 bosonic plus 8 $(4 \times 2)$ fermionic supertwistor variables subject to one bosonic constraint, Eq. (2.10). This mismatch indicates 
the presence of one bosonic and eight fermionic gauge symmetries in the superspace action (3.1).

The action is invariant under reparametrization as well as under the scaling (2.9), $\lambda^{\prime}=\lambda e^{\beta^{\prime}}$ (see Eq. (2.8)). Besides, there is a bosonic gauge symmetry under the $U(1)$ phase transformations of the spinor field $\lambda_{\alpha}$. This is the same gauge symmetry possessed by the supertwistor action (3.9), there generated by the first class constraint (2.10). Let us now show that the superspace action indeed possesses an 8-parametric fermionic $\kappa$-symmetry.

Varying the action (3.1) we find (mainly ignoring the Yang-Mills current part $L_{G}$ which does not depend neither on supertwistors nor on the superspace coordinate functions)

$$
\begin{array}{r}
\delta S=\int_{W^{2}} \delta e^{++} \wedge\left(\hat{\Pi}^{\dot{\alpha} \alpha} \bar{\lambda}_{\dot{\alpha}} \lambda_{\alpha}+\frac{\delta\left(d^{2} \xi L_{G}\right)}{\delta e^{++}}\right)- \\
-\int_{W^{2}} d\left(e^{++} \lambda_{\alpha} \bar{\lambda}_{\dot{\alpha}}\right)\left(\delta \hat{x}^{\dot{\alpha} \alpha}-i \delta \hat{\theta}_{i}^{\alpha} \hat{\bar{\theta}}^{\dot{\alpha} i}+i \hat{\theta}_{i}^{\alpha} \delta \hat{\bar{\theta}}^{\dot{\alpha} i}\right) \\
+\int_{W^{2}} e^{++} \wedge \hat{\Pi}^{\dot{\alpha} \alpha}\left(\bar{\lambda}_{\dot{\alpha}} \delta \lambda_{\alpha}+\delta \bar{\lambda}_{\dot{\alpha}} \lambda_{\alpha}\right) \\
-2 i \int_{W^{2}} e^{++} \wedge\left(d \theta_{i}^{\alpha} \lambda_{\alpha} \delta \bar{\theta}^{\dot{\alpha} i} \bar{\lambda}_{\dot{\alpha}}+d \bar{\theta}^{\dot{\alpha} i} \bar{\lambda}_{\dot{\alpha}} \delta \theta_{i}^{\alpha} \lambda_{\alpha}\right)
\end{array}
$$

The fact that only $8\left(\delta \theta_{i}^{\alpha} \lambda_{\alpha}\right.$ and its c.c. $)$ out of the $8+8$ independent fermionic variations $\left(\delta \theta_{i}^{\alpha}\right.$ and $\delta \bar{\theta}^{\dot{\alpha} i}=$ $\left.\left(\delta \theta_{i}^{\alpha}\right)^{*}, i=1,2,3,4\right)$ enter effectively in the action variation (3.11) shows that the action (3.1) possess eight local fermionic $\kappa$-symmetries. Explicitly they read

$$
\begin{gathered}
\delta_{\kappa} x^{\dot{\alpha} \alpha}=i \delta_{\kappa} \theta_{i}^{\alpha} \bar{\theta}^{\dot{\alpha} i}-i \theta_{i}^{\alpha} \delta_{\kappa} \bar{\theta}^{\dot{\alpha} i}, \\
\delta_{\kappa} \theta_{i}^{\alpha}=\kappa_{i} \lambda^{\alpha}, \quad \delta_{\kappa} \bar{\theta}^{\dot{\alpha} i}=\bar{\kappa}^{i} \bar{\lambda}^{\dot{\alpha}}, \\
\delta_{\kappa} \lambda^{\alpha}=\delta_{\kappa} \bar{\lambda}^{\dot{\alpha}}=\delta_{\kappa} e^{++}=0 .
\end{gathered}
$$

The $\kappa$-symmetry transformations in the form (3.12) are clearly irreducible (see [36] for their interpretation as worldline supersymmetry) in contrast with the standard $\kappa$-symmetry [37, 38] with

$$
\begin{aligned}
& \delta_{\kappa} \theta_{i}^{\alpha}=\kappa_{\dot{\alpha} i} \Pi_{--}^{\dot{\alpha} \alpha} \quad, \quad \delta_{\kappa} \bar{\theta}^{\dot{\alpha} i}=\Pi_{--}^{\dot{\alpha} \alpha} \bar{\kappa}_{\alpha}^{i} \\
& \left(\Pi_{--}^{\dot{\alpha} \alpha}:=\nabla_{--} x^{\dot{\alpha} \alpha}-i \nabla_{--} \theta^{\alpha} \bar{\theta}^{\dot{\alpha} \alpha}+i \theta^{\alpha} \nabla_{--} \bar{\theta}^{\dot{\alpha} \alpha}\right) .
\end{aligned}
$$

Clearly, the irreducible transformations (3.12) can be obtained from the standard $\kappa$-symmetry in its first order form by substituting $\bar{\lambda}^{\dot{\alpha}} \lambda^{\alpha}$ for $\Pi_{--}^{\dot{\alpha} \alpha}$ with $\kappa_{i}=\kappa_{i \dot{\alpha}} \bar{\lambda}^{\dot{\alpha}}$. Equation $\Pi_{--}^{\dot{\alpha} \alpha} \propto \bar{\lambda}^{\dot{\alpha}} \lambda^{\alpha}$ indeed holds on the mass shell for the dynamical system (3.1), see Eq. (3.7).

\section{The supertwistor string as a formulation of the tensionless string}

The fact that the action (3.9) corresponds to a tensionless superstring was noticed in [14]. We have seen above that the twistor string action (3.9) is equivalent to the superspace action (3.1) which includes the bosonic spinors $\lambda_{\alpha}$ as auxiliary variables. Our next observation is that the action (3.1) is simply the null-superstring action of [21, 22]. Indeed, although that action was written in terms of $D=4$ Lorentz harmonic variables $\left(v_{\alpha}^{-}, v_{\alpha}^{+}\right) \in S L(2, \mathbf{C})=\operatorname{Spin}(1,3)$, and (3.1) contains instead one bosonic spinor $\lambda_{\alpha}$, the second harmonic $v_{\alpha}^{+}$ was not involved in the null-superstring action of [21, 22]. Furthermore, the only constraint that is imposed on these $D=4$ spinorial harmonics [33] is

$$
v^{\alpha-} v_{\alpha}^{+}=1
$$

If this is considered as a condition on $v_{\alpha}^{+}$, then $v_{\alpha}^{-}$is just an arbitrary but nonvanishing bosonic spinor and can be identified with $\lambda_{\alpha}$. Then, with $\lambda_{\alpha}=v_{\alpha}^{-}$, and defining a worldsheet density $\rho^{++m}$ by $e^{++} \wedge d:=d^{2} \xi \rho^{++m} \partial_{m}$, one can write the action (3.1) in the form

$$
S=\int_{W^{2}} d^{2} \xi \rho^{++m} \Pi_{m}^{\dot{\alpha} \alpha} \bar{v}_{\dot{\alpha}}^{-} v_{\alpha}^{-} \equiv \int_{W^{2}} e^{++} \wedge \Pi^{\dot{\alpha} \alpha} \bar{v}_{\dot{\alpha}}^{-} v_{\alpha}^{-}
$$

which is exactly the 'twistor-like' tensionless superstring action in 21, 22].

\section{Berkovits supertwistor open string model and the open tensionless superstring in an enlarged superspace}

The above observations indicate that Berkovits open string version of the twistor string model should correspond to the open null-string. We show now that this open tensionless superstring can be defined on the direct product of two $N=4$ superspaces [66].

Let us consider the left and right moving supertwistors already restricted by the constraints

$$
\begin{gathered}
\bar{\Upsilon}_{\Sigma}^{-} \Upsilon^{-\Sigma} \equiv \bar{\lambda}_{\dot{\alpha}}^{-} \mu^{-\dot{\alpha}}-\bar{\mu}^{-\alpha} \lambda_{\alpha}^{-}-2 i \eta_{i}^{-} \bar{\eta}^{-i}=0, \\
\bar{\Upsilon}_{\Sigma}^{+} \Upsilon^{+\Sigma} \equiv \bar{\lambda}_{\dot{\alpha}}^{+} \mu^{+\dot{\alpha}}-\bar{\mu}^{+\alpha} \lambda_{\alpha}^{+}-2 i \eta_{i}^{+} \bar{\eta}^{+i}=0 .
\end{gathered}
$$

Then the connection $B=e^{++} B_{++}+e^{--} B_{--}$(which in the original formulation of Eq. (2.14) 12] reproduces the above two constraints as the Euler-Lagrange equations for $B_{--}$and $\left.B_{++}\right)$disappears from the action, which takes the form

$$
\begin{aligned}
S_{B}= & \int_{W^{2}}\left[e^{++} \wedge \bar{\Upsilon}_{\Sigma}^{-} d \Upsilon^{-\Sigma}-e^{--} \wedge \bar{\Upsilon}_{\Sigma}^{+} d \Upsilon^{+\Sigma}\right]+ \\
& +\int_{W^{2}} d^{2} \xi L_{G}
\end{aligned}
$$

The local Lorentz symmetry $S O(1,1)$ of the action (3.18) as well as its two $U(1)$ gauge symmetries, one acting on $\Upsilon^{+\Sigma}$ and $\bar{\Upsilon}_{\Sigma}^{+}$and the other on $\Upsilon^{-\Sigma}$ and $\bar{\Upsilon}_{\Sigma}^{-}$, hold true due to the constraints (3.16), (3.17) imposed on $\Upsilon^{-}$and $\Upsilon^{+}$. The action also has an overall scaling gauge symmetry under $\Upsilon^{+,-} \mapsto e^{\gamma} \Upsilon^{+,-}, e^{ \pm \pm} \mapsto e^{-2 \gamma} e^{ \pm \pm}$.

Now, following with this action the same steps as in Sec. IIIA, but in reverse order, we recover a counterpart of (3.1) for the open twistor string action of Eq. 
(2.14). Starting from Eq. (3.18) and solving the constraints (3.16), (3.17) by

$$
\begin{aligned}
\mu^{-\dot{\alpha}} & =x_{(l) L}^{\dot{\alpha} \alpha} \lambda_{\alpha}^{-}:=\left(x_{(l)}^{\dot{\alpha} \alpha}+i \theta_{(l) i}^{\alpha} \bar{\theta}_{(l)}^{\dot{\alpha} i}\right) \lambda_{\alpha}^{-}, \\
\eta_{i}^{-} & =\theta_{(l) i}{ }_{i} \lambda_{\alpha}^{-} ; \\
\mu^{+\dot{\alpha}} & =x_{(r) L}^{\dot{\alpha} \alpha} \lambda_{\alpha}^{+}:=\left(x_{(r)}^{\dot{\alpha} \alpha}+i \theta_{(r) i}^{\alpha} \bar{\theta}_{(r)}^{\dot{\alpha} i}\right) \lambda_{\alpha}^{+}, \\
\eta_{i}^{+} & =\theta_{(r) i}{ }_{i} \lambda_{\alpha}^{+},
\end{aligned}
$$

one finds that the action $S_{B}$ of (3.18) is equivalent to

$$
\begin{aligned}
S= & \int_{W^{2}}\left(e^{++} \wedge \hat{\Pi}_{(l)}^{\dot{\alpha} \alpha} \bar{\lambda}_{\dot{\alpha}}^{-} \lambda_{\alpha}^{-}-e^{--} \wedge \hat{\Pi}_{(r)}^{\dot{\alpha} \alpha} \bar{\lambda}_{\dot{\alpha}}^{+} \lambda_{\alpha}^{+}\right) \\
& +\int_{W^{2}} d^{2} \xi L_{G},
\end{aligned}
$$

where

$$
\begin{aligned}
& \Pi_{(l)}^{\dot{\alpha} \alpha}:=d x_{(l)}^{\dot{\alpha} \alpha}-i d \theta_{(l) i}^{\alpha} \bar{\theta}_{(l)}^{\dot{\alpha} i}+i \theta_{(l) i}^{\alpha} d \bar{\theta}_{(l)}^{\dot{\alpha} i}, \\
& \Pi_{(r)}^{\dot{\alpha} \alpha}:=d x_{(r)}^{\dot{\alpha} \alpha}-i d \theta_{(r) i}^{\alpha} \bar{\theta}_{(r)}^{\dot{\alpha} i}+i \theta_{(r) i}^{\alpha} d \bar{\theta}_{(r)}^{\dot{\alpha} i} .
\end{aligned}
$$

The action (3.21) contains two sets of coordinate functions, $x_{(l)}^{\alpha \dot{\alpha}}, \theta_{(l) i}^{\alpha}, \bar{\theta}_{(l)}^{\dot{\alpha} i}$ and $x_{(r)}^{\alpha \dot{\alpha}}, \theta_{(r) i}^{\alpha}, \bar{\theta}_{(r)}^{\dot{\alpha} i}$, corresponding to two copies of $D=4, N=4$ superspace. Looking at the dynamics implied by (3.21) it is seen that one set $\left(x_{(l)}^{\alpha \dot{\alpha}}, \theta_{(l) i}^{\alpha}, \bar{\theta}_{(l)}^{\dot{\alpha} i}\right)$ contains the left- and the other $\left(x_{(r)}^{\alpha \dot{\alpha}}, \theta_{(r) i}^{\alpha}, \bar{\theta}_{(r)}^{\dot{\alpha} i}\right)$ the right-moving fields, as indicated by the subindexes $l, r$.

We note in passing that such a double set of variables, but for $N=1$, was used in [23] to write an equivalent form of the $N=2$ Green-Schwarz superstring action.

In the above discussion on the open twistor string, the doubling of the superspace variables seems to play an auxiliary rôle as far as the YM vertex operators are associated with the boundary of the open string. The two sets of $D=4 N=4$ superspace coordinate functions are needed to formulate the action in its spacetime form. The boundary conditions (2.15) identify these coordinate functions modulo the (two copies of the) $\kappa$-symmetry,

$$
\begin{gathered}
\delta_{\kappa} x_{(l)}^{\dot{\alpha} \alpha}=i \delta_{\kappa} \theta_{(l) i}^{\alpha} \bar{\theta}_{(l)}^{\dot{\alpha} i}-i \theta_{(l) i}^{\alpha} \delta_{\kappa} \bar{\theta}_{(l)}^{\dot{\alpha} i}, \\
\delta_{\kappa} \theta_{(l) i}^{\alpha}=\kappa_{i}^{+} \lambda^{-\alpha}, \quad \delta_{\kappa} \bar{\theta}_{(l)}^{\dot{\alpha} i}=\bar{\kappa}^{+i} \bar{\lambda}^{-\dot{\alpha}}, \\
\delta_{\kappa} x_{(r)}^{\dot{\alpha} \alpha}=i \delta_{\kappa} \theta_{(r) i}^{\alpha} \bar{\theta}_{(r)}^{\dot{\alpha} i}-i \theta_{(r) i}^{\alpha} \delta_{\kappa} \bar{\theta}_{(r)}^{\dot{\alpha} i}, \\
\delta_{\kappa} \theta_{(r) i}^{\alpha}=\kappa_{i}^{-} \lambda^{+\alpha}, \quad \delta_{\kappa} \bar{\theta}_{(r)}^{\dot{\alpha} i}=\bar{\kappa}^{-i} \bar{\lambda}^{+\dot{\alpha}}, \\
\delta_{\kappa} \lambda^{ \pm \alpha}=\delta_{\kappa} \bar{\lambda}^{ \pm \dot{\alpha}}=\delta_{\kappa} e^{ \pm \pm}=0,
\end{gathered}
$$

and reparametrization symmetry transformations characteristic of the action (3.21).

\section{ON A POSSIBLE PARENT TENSIONFUL SUPERSTRING ACTION FOR THE TWISTOR STRING}

The null-superstring mass spectrum is known to be continuous 22, 24]. To obtain a discrete spectrum, one should rather quantize the tensionless limit of a tensionful superstring. These two zero tension superstrings are different in the set of variables used to build the quantum theory. The null-superstring [22, 24] is quantized in terms of particle-like variables, momentum and coordinates, while the quantum theory of the tensionless limit of a superstring (often called just 'tensionless superstring') is formulated in terms of stringy oscillators [26, 27, 41].

The calculations of the tree YM diagrams from the twistor string models 1, 12, 14], in particular the choice of the vertex operators and the discussions on contributions to conformal anomaly, clearly use stringy variables rather than the particle-like null-superstring ones and, thus, deal with a tensionless limit of some tensionful superstring rather than with the intrinsically tensionless superstring i.e. null-superstring.

Thus it is natural to ask: which is the tensionful superstring action the tensionless limit of which leads to the twistor string one? Such a problem was posed by Siegel [14], who proposed the tensionful QCD string [28] as the bosonic part of such a parent superstring; fermions were not considered. In our present perspective, the above problem corresponds to looking for the tensionful parent of the $N=4$ version of the $N=1$ tensionless superstring action of [21, 22], Eq. (3.15).

\section{A. From tensionful $\mathrm{D}=4$ superstrings to $\mathrm{N}=1,2$ counterparts of the supertwistor string}

The $N=1$ and $N=2$ versions of the null-superstring superspace action (3.15) can be obtained as tensionless limits of the action of the Lorentz harmonics formulation [42] of the $N=1$ and $N=2 D=4$ Green-Schwarz superstrings

$$
S=\frac{1}{4 \pi \alpha^{\prime}} \int_{W^{2}}\left[e^{++} \wedge \Pi^{\dot{\alpha} \alpha} \bar{v}_{\dot{\alpha}}^{-} v_{\alpha}^{-}-e^{--} \wedge \Pi^{\dot{\alpha} \alpha} \bar{v}_{\dot{\alpha}}^{+} v_{\alpha}^{+}-e^{++} \wedge e^{--}\right]-\frac{1}{4 \pi \alpha^{\prime}} \int_{W^{2}} \widehat{B}_{2},
$$

where the bosonic spinors $v_{\alpha}^{-}$and $v_{\alpha}^{+}$are relatively normalized by the 'harmonicity conditions' (3.14), $v^{\alpha-} v_{\alpha}^{+}=1$, and the last contribution in 4.1) is the Wess- 
Zumino term. This is defined by the pull-back to $W^{2}$ of the two-form gauge potential $B_{2}$ on flat superspace and that provides the superspace generalization of the NS-NS or Kalb-Ramond field. This two-form obeys the constraints [67]

$$
\begin{gathered}
H_{3}=d B_{2}=-2 i \Pi^{a} \wedge\left(d \theta^{1} \wedge \sigma_{a} d \bar{\theta}^{1}-d \theta^{1} \wedge \sigma_{a} d \bar{\theta}^{2}\right) \\
\qquad \text { for } N=2 \\
H_{3}=d B_{2}=-2 i \Pi^{a} \wedge d \theta \wedge \sigma_{a} d \bar{\theta} \quad \text { for } N=1
\end{gathered}
$$

The $N=1,2$ versions of the null-superstring action (3.15) can be obtained from (4.1) by taking the tensionless limit $\alpha^{\prime} \mapsto \infty$ while keeping $\frac{e^{++}}{\alpha^{\prime}}$ finite. Thus, before setting $\alpha^{\prime} \mapsto \infty$ we redefine $e^{++} \rightarrow 4 \pi \alpha^{\prime} e^{++}$, $e^{--} \rightarrow e^{--} /\left(4 \pi \alpha^{\prime}\right)$. In this way, taking the tensionless limit $\alpha^{\prime} \mapsto \infty$ one finds that the Wess-Zumino term and the 'cosmological' $e^{++} \wedge e^{--}$term vanish as $1 / \alpha^{\prime} \mapsto 0$. Similarly, the second term in (4.1) also goes to zero as $1 /\left(\alpha^{\prime}\right)^{2} \mapsto 0$, while after the redefinition the first term becomes $\alpha^{\prime}$ independent and produces the tensionless superstring action (3.15).

The problem with the $N=4$ tensionless superstring, which is equivalent to the twistor string model 1, 12, 14], is that the corresponding $N=4$ tensionful superstring, which would be the counterpart of the $N=1,2$ ac- tions (4.1) possessing a $2 N$ parametric $\kappa$-symmetry, is not known. This problem may be traced to the mismatch between the on-shell bosonic and fermionic degrees of freedom of such a hypothetical $N=4$ superstring constructed from $\hat{x}^{a}, \hat{\theta}^{\alpha 1}, \ldots, \hat{\theta}^{\alpha 4}$ and their complex conjugates $(4-2=2$ bosonic and $1 / 2(4 \times 2)=4$ fermionic degrees of freedom). Geometrically, the problem is reflected by the absence of the $D=4, N=4$ counterpart of the CE three-cocycles $H_{3}=d B_{2}$ [4] that do exist in $D=4, N=1,2$ superspaces. Such a closed three form would be needed to construct the Wess-Zumino term, a necessary ingredient of a $\kappa$-symmetric tensionful superstring action in the superspace of the usual type (see 44] for a superstring action without Wess-Zumino term in an enlarged tensorial superspace and [4] for a discussion of WZ terms and extended superspaces).

\section{B. From $\mathrm{D}=10 \mathrm{~N}=1$ superstring to the supertwistor string}

Such a three-cocycle does exist for the $D=10 N=1$ supersymmetry algebra, allowing for the existence of the heterotic superstring [46]. It is given by

$$
D=10, N=1: \quad H_{3}=d B_{2}=-2 i \Pi \underline{a} \wedge d \Theta \wedge \Sigma_{\underline{a}} d \Theta .
$$

The $N=1 D=10$ superstring contains (as the $D=4, N=4$ one) 16 fermionic Majorana-Weyl coordinate functions

$$
\Theta^{\underline{\alpha}}=\left(\begin{array}{c}
\theta_{i}^{\alpha} \\
\bar{\theta}_{\dot{\alpha}}^{i}
\end{array}\right) \equiv\left(\begin{array}{c}
\theta_{i}^{\alpha} \\
\left(\theta_{\alpha i}\right)^{*}
\end{array}\right), \quad \underline{\alpha}=1, \ldots, 16, \quad \alpha=1,2, \quad \dot{\alpha}=1,2, \quad i=1, \ldots 4,
$$

and ten bosonic coordinate functions $X^{\underline{a}}$ which can be split as

$$
X^{\underline{a}}=\left(x^{a}, X^{I}\right), \quad \underline{a}=0,1 \ldots, 10, \quad a=0,1,2,3, \quad I=1, \ldots, 6 .
$$

The $D=10(16 \times 16)$ sigma-matrices can be chosen in the form

$$
\Sigma_{\alpha \beta}^{\underline{a}}=\left(\Sigma_{\alpha \beta}^{a}, \Sigma_{\alpha \beta}^{I}\right), \quad \Sigma_{\alpha \beta}^{a}=\left(\begin{array}{cc}
0 & \sigma_{\alpha \dot{\beta}}^{a} \delta_{j}^{i} \\
\sigma^{a \dot{\alpha} \beta} \delta_{i}^{j} & 0
\end{array}\right), \quad \Sigma_{\alpha \beta}^{I}=\left(\begin{array}{cc}
\epsilon_{\alpha \beta} \tilde{\rho}^{I i j} & 0 \\
0 & -\epsilon_{\dot{\alpha} \dot{\beta}} \rho_{i j}^{I}
\end{array}\right),
$$

where $\rho_{i j}^{I}$ and $\tilde{\rho}^{I i j}$ are the $S O(6)$ Clebsch-Gordan coefficients $\rho_{i j}^{I} \tilde{\rho}^{I i^{\prime} j^{\prime}}=-4 \delta_{i}^{\left[i^{\prime}\right.} \delta_{j}^{\left.j^{\prime}\right]}$ (see e.g. [47]).

The Lorentz harmonics formulation of the $D=10, N=1$ superstring is characterized by the action [42, 48] which can be written in the form

$$
S=\frac{1}{4 \pi \alpha^{\prime}} \int_{W^{2}}\left[e^{++} \wedge \Pi \underline{a}_{\underline{a}^{-}}{ }^{--}-e^{--} \wedge \Pi \underline{a}_{\underline{a}^{\prime}}{ }^{++}-e^{++} \wedge e^{--}\right]-\frac{1}{4 \pi \alpha^{\prime}} \int_{W^{2}} \hat{B}_{2}
$$

involving the worldvolume fields in the pull-back of the NS-NS two form $B_{2}$ (4.4) to $W^{2}$ and two auxiliary lightlike vector fields, $u_{\underline{a}}^{++}, u_{\underline{a}}^{--}$, the counterparts of $v_{\alpha}^{-} \bar{v}_{\dot{\alpha}}^{-}$and $v_{\alpha}^{+} \bar{v}_{\dot{\alpha}}^{+}$in $D=4$ (4.1) [68]. These 'vector Lorentz harmonics' [50] may be considered as composites of the $D=10$ spinorial harmonics or spinor moving frame variables [42, 48, 51]. Here we only notice their lightlike character and the relative normalization (cf. (3.14)) of the ten-vectors $u_{\underline{a}}^{ \pm \pm}$,

$$
u^{\underline{a}--} u_{\underline{a}}{ }^{--}=0, \quad u^{\underline{a}++} u_{\underline{a}}{ }^{++}=0, \quad u^{\underline{a}--} u_{\underline{a}}{ }^{++}=2 .
$$


Taking the $\alpha^{\prime} \mapsto \infty$ limit in Eq. (4.8) after the $e^{++} \rightarrow 4 \pi \alpha^{\prime} e^{++}, e^{--} \rightarrow e^{--} /\left(4 \pi \alpha^{\prime}\right)$ redefinition, as for the $D=4, N=1,2$ superstring action above, we arrive at the ten-dimensional tensionless superstring action

$$
S=\int_{W^{2}} e^{++} \wedge \Pi^{\underline{a}} u_{\underline{a}}^{--}, \quad u_{\underline{a}}^{--} u^{\underline{a}--}=0,
$$

which involves only $u_{\underline{a}}^{--}$, one of the two lightlike tendimensional vectors (4.9). A dimensional reduction of such an action can be done in such a manner that the $D=4, N=4$ null superstring appears. A formal way to achieve this is to consider the action (4.10) in a frame where the above lightlike vector $u_{\underline{a}}^{--}$only has nonvanishing components in the four $D=\overline{4}$ Minkowski spacetime directions,

$$
\begin{gathered}
u_{\underline{a}}^{--}=\delta_{\underline{a}}^{b} u_{b}^{--}=\left(u_{a}^{--}, 0, \ldots, 0\right), \quad u_{a}^{--} u^{a--}=0, \\
\underline{a}=0, \ldots, 9, \quad a=0, \ldots, 3 .
\end{gathered}
$$

\section{Tensorial (enlarged) superspace versus standard ten dimensional superspace.}

The above shows that the twistor string can be obtained by taking the tensionless limit of the $D=10 \mathrm{su}-$ perstring action (4.8) and then performing a dimensional reduction down to $D=4$. By considering the $D=10$ Green-Schwarz superstring action as a tensionful parent of the $D=4$ twistor string, we have allowed ourselves to enlarge $D=4$ superspace by six additional bosonic coordinates. However, it is not clear at present whether this enlargement is unique, and this allows us to discuss another possible higher-dimensional superstring parent for the twistor string.

Indeed, even if we restrict ourselves to just six additional bosonic coordinates as above, these do not need being the components of the $S O(6)$ vector $X^{I}$ implied in the enlargement of $D=4$ to the standard $D=10$ superspace. We may consider instead a tensorial superspace, in which the additional six bosonic coordinates appear as the components of an antisymmetric tensor, $Y^{\mu \nu}=-Y^{\nu \mu}$. The proper incorporation of a $\mathrm{SO}(6)$ vector into the action leads naturally to an enhancement of the symmetry from $S O(1,3) \otimes S O(6)$ to $S O(1,9)$. This implies an embedding of our tensionless string (classically equivalent to the twistor string) into a manifestly $S O(1,9)$ (actually, $D=10$ super-Poincaré) invariant theory. Similarly, the proper enlargement of the target superspace by the antisymmetric tensor coordinates $Y^{\mu \nu}$, which could be split into the symmetric spin-tensor $X^{\alpha \beta}=X^{\beta \alpha}$ and its complex conjugate (in the case of Minkowski signature) $X^{\dot{\alpha} \dot{\beta}}=X^{\dot{\beta} \dot{\alpha}}=\left(X^{\alpha \beta}\right)^{*}$, results in an enlargement of the automorphism symmetry to $G L(4, \mathbb{R})$. The spin-tensorial representation allows to collect all ten coordinates in a manifestly symmetric $4 \times 4$ matrix,

$$
\mathbb{X}^{\alpha^{\prime} \beta^{\prime}}:=\left(\begin{array}{cc}
X^{\alpha \beta} & \left(X^{\dot{\beta} \alpha}\right)^{T} \\
X^{\dot{\alpha} \beta} & X^{\dot{\alpha} \dot{\beta}}
\end{array}\right), \quad \alpha^{\prime}, \beta^{\prime}=1, \ldots, n=4
$$

(i.e. $\left.\alpha^{\prime}=1,2, \dot{1}, \dot{2}\right)$. Such a tensorial space was proposed by Fronsdal [52 to describe higher spin fields. A dynamical realization of such a theory was found later 53., quantizing a generalized superparticle model 54 which has the properties of a BPS preon [55] (see [44, 56, 57] for further discussion).

The above analysis suggests relating our $D=4, N=4$ null-superstring (3.15) to a string model in a $N=4$ extended tensorial superspace $\widetilde{\Sigma}^{(10 \mid 4 N)}\left(=\widetilde{\Sigma}^{\left(\frac{n(n+1)}{2} \mid n N\right)}\right.$ for $n=4$, see [4])

$$
\left(\mathbb{X}^{\alpha^{\prime} \beta^{\prime}}, \Theta^{\alpha^{\prime} i}\right):=\left(X^{\dot{\beta} \beta}, X^{\alpha \beta}, X^{\dot{\alpha} \dot{\beta}} ; \theta_{i}^{\alpha}, \bar{\theta}^{\dot{\alpha} i}\right) .
$$

The $D=4, N=4$ null superstring action (3.15) providing a spacetime reformulation of the Berkovits-Siegel twistor string action (3.1) can be obtained as the $w \mapsto 0$ limit of the action (omitting the $L_{G}$ contribution)

$$
\begin{gathered}
S(w)=\int_{W^{2}} e^{++} \wedge\left(\Pi^{\dot{\alpha} \alpha} \lambda_{\alpha} \bar{\lambda}_{\dot{\alpha}}+\frac{w}{2} \Pi^{\alpha \beta} \lambda_{\alpha} \lambda_{\beta}+\frac{\bar{w}}{2} \Pi^{\dot{\alpha} \dot{\beta}} \bar{\lambda}_{\dot{\alpha}} \bar{\lambda}_{\dot{\beta}}\right), \\
\Pi^{\dot{\alpha} \alpha}=d x^{\dot{\alpha} \alpha}-i d \theta_{i}^{\alpha} \bar{\theta}^{\dot{\alpha} i}+i \theta_{i}^{\alpha} d \bar{\theta}^{\dot{\alpha} i}, \quad \Pi^{\alpha \beta}=d x^{\alpha \beta}-2 i d \theta_{i}^{(\alpha} \theta_{i}^{\beta)}, \quad \Pi^{\dot{\alpha} \dot{\beta}}=d x^{\dot{\alpha} \dot{\beta}}-2 i d \bar{\theta}^{(\dot{\alpha} i} \bar{\theta}^{\dot{\beta}) i},
\end{gathered}
$$

which describes a tensionless superstring in $N=4$ extended tensorial superspace $\widetilde{\Sigma}^{(10 \mid 4 N)}$ (for $w=1$ this ac- tion was first considered in [40]).

The action (4.14), is an extended object counterpart 
of the superparticle action [53] in tensorial superspace. It may be related with the tensionless limit of tensionful superstring models in enlarged superspace (higher spin extensions of the superstring) considered in [4]]. In particular, a direct tensionless limit of the generalized superstring model [44] would lead to the $w=1$ representative of the family (4.14) of tensionless actions. This $w=1$ action can be rewritten in the form

$$
\begin{aligned}
S(w=1)= & S_{S p(4 \mid 4)}=\frac{1}{2} \int_{W^{2}} e^{++} \wedge \Pi^{\alpha^{\prime} \beta^{\prime}} \Lambda_{\alpha^{\prime}} \Lambda_{\beta^{\prime}}, \\
\Pi^{\alpha^{\prime} \beta^{\prime}} & =d \mathbb{X}^{\alpha^{\prime} \beta^{\prime}}-2 i d \Theta^{\left(\alpha^{\prime} \mid i\right.} \Theta^{\left.\mid \beta^{\prime}\right) i} \\
\Theta^{\alpha^{\prime} i} & :=\left(\theta_{i}^{\alpha}, \bar{\theta}^{\dot{\alpha} i}\right), \quad \Lambda_{\alpha^{\prime}}:=\left(\lambda_{\alpha}, \bar{\lambda}_{\dot{\alpha}}\right)
\end{aligned}
$$

which makes its $G L(4, \mathbb{R})$ symmetry manifest. It possesses a hidden $O S p(N \mid 8)$ symmetry which becomes manifest in its orthosymplectic twistor presentation 58

$$
\begin{gathered}
S_{\Sigma^{(10 \mid 16)}}=\frac{1}{2} \int e^{++} \wedge\left(d \mathcal{M}^{\alpha^{\prime}} \Lambda_{\alpha^{\prime}}-\mathcal{M}^{\alpha^{\prime}} d \Lambda_{\alpha^{\prime}}-2 i d \chi^{i} \chi^{i}\right) \\
\mathcal{M}^{\alpha^{\prime}}=\mathbb{X}^{\alpha^{\prime} \beta^{\prime}} \Lambda_{\beta^{\prime}}-i \Theta^{\alpha^{\prime} i} \Theta^{\beta^{\prime} i} \Lambda_{\beta^{\prime}} \\
\chi^{i}=\Theta^{\beta^{\prime} i} \Lambda_{\beta^{\prime}}
\end{gathered}
$$

(see 53, 54] for the superparticle case and the discussion in [44]).

In the purely bosonic limit the simple redefinition $\hat{X}^{\alpha \beta} \mapsto 1 / w \hat{X}^{\alpha \beta}, \hat{X}^{\dot{\alpha} \dot{\beta}} \mapsto 1 / \bar{w} \hat{X}^{\dot{\alpha} \dot{\beta}}$ maps any $w \neq 0$ model to the $w=1$ one. This implies that the symmetry of any of the $S(w \neq 0)$ actions (4.14) includes the bosonic $S p(8)$ group. However, the presence of fermions breaks this identification and makes the $w=1 \mathrm{dy}$ namical system (4.16) special as it possesses 12 local fermionic $\kappa$-symmetries while all other $w \neq 0,1$ models possess only $8 \kappa$-symmetries. Another face of the same fact is that the $w=1$ model (4.16) may be written in terms of $O S p(N \mid 8)=O S p(4 \mid 8)$ real supertwistors $\left(\mu^{\alpha^{\prime}}, \lambda_{\alpha^{\prime}}, \chi_{i}\right)$ with real fermionic $\chi_{i}=\left(\chi_{i}\right)^{*} i=$ $1, \ldots, 4$, Eqs. (4.17), while the $w \neq 0,1$ models require $\operatorname{OSp}(2 N \mid 8)=\operatorname{OSp}(8 \mid 8)$ supertwistors $\left(\mu^{\alpha^{\prime}}, \lambda_{\alpha^{\prime}}, \eta_{i}\right)$ with complex fermionic components $\eta_{i} \neq\left(\eta_{i}\right)^{*}$ (see [53] for the superparticle case),

$$
S(w)=\frac{1}{2} \int e^{++} \wedge\left(d \bar{\mu}^{\alpha} \lambda_{\alpha}-d \lambda_{\alpha} \bar{\mu}^{\alpha}+\bar{\lambda}_{\dot{\alpha}} d \mu^{\dot{\alpha}}-\mu^{\dot{\alpha}} d \bar{\lambda}_{\dot{\alpha}}-2 i d \eta_{i}\left(\bar{\eta}^{i}+w \eta_{i}\right)+2 i\left(\eta_{i}+\bar{w} \bar{\eta}^{i}\right) d \bar{\eta}^{i}\right)
$$

where

$$
\begin{gathered}
\bar{\mu}^{\alpha}=X^{\dot{\beta} \alpha} \bar{\lambda}_{\dot{\beta}}+w X^{\alpha \beta} \lambda_{\beta}-i \theta_{i}^{\alpha}\left(\bar{\theta}^{i} \bar{\lambda}+w \theta_{i} \lambda\right), \quad \mu^{\dot{\alpha}}=X^{\dot{\alpha} \beta} \lambda_{\beta}+\bar{w}^{\dot{\alpha} \dot{\beta}} \bar{\lambda}_{\dot{\beta}}-i \bar{\theta}^{\dot{\alpha} i}\left(\theta_{i} \lambda+\bar{w} \bar{\theta}^{i} \bar{\lambda}\right), \\
\eta_{i}=\theta_{i} \lambda=\theta_{i}^{\alpha} \lambda_{\alpha}, \quad \bar{\eta}^{i}=\bar{\theta}^{i} \bar{\lambda}=\bar{\theta}^{\dot{\alpha} i} \bar{\lambda}_{\dot{\alpha}} .
\end{gathered}
$$

The $w=0$ member of the above $S(\omega)$ family is equivalent to the twistor string action (2.1),

$$
S(w=0)=S_{S}=\int_{W^{2}} e^{++} \wedge \Pi^{\dot{\alpha} \alpha} \lambda_{\alpha} \bar{\lambda}_{\dot{\alpha}}
$$

To see this, in addition to observing the coincidence of Eqs. (3.1) and (4.20), one should take into account that for $w=0$ Eqs. (4.19) become the incidence equations (3.10) and their c.c.; these, in turn, provide the general solution of the constraints (2.10). For the general $w \neq 0$ element of the family of dynamical systems (4.18), Eqs. (4.19) do not imply any constraints. This corresponds to the fact that the $N=1$ superparticle counterpart of the action (4.18) describes an infinite tower of massless fields of all possible helicities ('free higher spin theory'). The significance of the fact that the twistor string enters as a singular element of the one-complex-parameter family of tensionless superstrings in tensorial superspace (if any) is still to be understood. Let us finish by noticing that there is an enlargement of the internal symmetry group of the $w=0$ action (4.18) from $S O(4)$ to $S U(4)$.

\section{FINAL REMARKS AND DISCUSSION}

By using a twistor transform similar to the one originally proposed for the superparticle [19], we have seen that the twistor string model is classically equivalent to a supersymmetric extended object in a $D=4, N=4$ superspace. For Siegel's closed string version of the Berkovits model, this action, having $8 \kappa$-symmetries and 16 supersymmetries, coincides with the $N=4$ extension of the tensionless superstring action 21]. The Berkovits open-string action [12] describes a counterpart of the tensionless superstring in the superspace isomorphic to a direct product of two copies of $D=4, N=4$; the two copies of the coordinate functions turn out to be identified on the open string boundary modulo gauge symmetries, the set of which includes two copies of 8-parametric $\kappa$-symmetry, Eqs. (3.23), (3.24).

Null (or intrinsically tensionless) superstrings maintain their conformal invariance by having a continuous spectrum [21, 24]. This implies that the prescription of writing the gauge field amplitudes from [14] assumes dealing with the tensionless limit of some tensionful superstring rather than with the null-superstring itself. This was 
actually noticed in 14 where a possible relation of the twistor superstring with a model for a 'QCD string' 28 was discussed. However, the consideration of the tensionful prototype of the twistor string in 14] was purely bosonic.

It is plausible to assume that a parent tensionful superstring should have a smooth tensionless superstring limit in the sense that both tensionful and tensionless superstrings should present the same number of $\kappa$-symmetries. Of course, as it is known, the zero tension limit is special in many respects. In particular the huge enhancement of the global symmetry in this limit was already noticed in 25. However, one may expect a nonsingular limit in the sense of preserving the number of degrees of freedom of the dynamical system. This seems to be the case for the tensionless limit of the standard (Nambu-Goto or) Green-Schwarz string, a limit believed to be described by a massless higher spin theory (an infinite tower of massless higher spin fields); see [27, 41] for a discussion. Let us stress, nevertheless, that the tensionless limit of the usual Green-Schwarz superstring leading to the twistor string is expected to be different. Such a zero tension limit could accompanied by some other transformations of the variables in the model. In any case, since Berkovits and Siegel's path integral exponents contain the twistor string action and we have seen here that this action is equivalent to the tensionless string one, our results suggest that their prescription to obtain the $N=4 \mathrm{SYM}$ diagrams from the twistor string [12, 14] provides a third way of quantizing of the tensionless string, alternative to the two of [26, 27], 21, 22].

As we discussed in this paper, the $D=10 N=1$ superstring can be considered as a tensionful candidate leading to the $D=4$ twistor string upon dimensional reduction. At the present level of understanding, the way from $D=10 N=1$ tensionful Green-Schwarz superstring to the $D=4 N=1$ tensionless superstring action (3.15) (equivalent, as we have shown here, to Siegel's twistor string action (2.1)), consists in taking first a tensionless limit then performing a dimensional reduction of the $D=10$ tensionless superstring down to $D=4$. For such a construction the standard $D=10 N=1 \mathrm{su}-$ perspace $\Sigma^{(10 \mid 16)}$ is not a priori a better starting point than e.g., $D=4 N=4$ tensorial superspace $\widetilde{\Sigma}^{(10 \mid 16)}$, the ten bosonic coordinates of which include the spacetime four-vector $x^{\mu}$ plus six tensorial coordinates $y^{\mu \nu}$. These can be treated as spin degrees of freedom [52, 53. or as conjugated 44, 45, 54] to the topological charges of superbranes [59].

This is a good place to discuss the possible higher dimensional generalizations of the supertwistor string (a problem also posed in [16] in the context of twotime physics). The generalization to tensorial superspace $\widetilde{\Sigma}^{(10 \mid 16)}$ can be associated with any of the tensionless superstring actions (4.14) with $w \neq 0$. The pure twistor form of the action similar to the Berkovits-Siegel one for a supertwistor string is provided by Eq. (4.18). A possible drawback of this action is the lack of a complex struc- ture and hence of a $U(1)$ symmetry, which seems relevant in applying the supertwistor string to Yang-Mills theory 1, 12, 14], although one cannot exclude the (rather exotic) possibility of replacing this $U(1)$ symmetry of the $w=0$ action by some other symmetry of the $w \neq 0$ models. The same lack of complex structure results in a replacement of the $S U(2,2 \mid 4)$ superconformal symmetry of the $w=0$ action by the $O S p(8 \mid 8)$ generalized conformal symmetry of the $S(w \neq 0)$ models 4.18) $(O S p(4 \mid 8)$ for $w=1$ ).

The generalization of the twistor superstring to the more conventional $D=10 N=1$ superspace is actually provided by the tensionless superstring action 4.10). To see this one needs, following [42, 48], to 'extract the square root' of the light-like vector $u_{\underline{a}}^{--}$(vector Lorentz harmonics) by introducing a set of 8 bosonic spinors $v_{\underline{\alpha}} \bar{q}$ (basis of the spinor moving frame or spinor Lorentz harmonics) highly constrained by

$$
\begin{gathered}
2 v_{\underline{\alpha} q}^{-} v_{\underline{\beta} q}^{-}=u_{\underline{a}}^{--} \Sigma_{\underline{\alpha} \underline{\underline{\beta}}}^{-}, \quad v_{p}^{-} \tilde{\Sigma}_{\underline{a}} v_{q}^{-}=\delta_{p q} u_{\underline{a}}^{--} \\
\underline{\alpha}, \underline{\beta}=1, \ldots, 16, \quad p, q=1, \ldots, 8
\end{gathered}
$$

$\left(\Sigma \underline{\underline{a}} \widetilde{\underline{b}} \underline{\underline{b}}+\Sigma^{\underline{b}} \widetilde{\Sigma} \underline{a}=2 \eta \underline{\underline{a b}}\right.$ and $\Sigma^{\underline{a}}$ was defined in (4.7)). Then, the action (4.10) reads

$$
\begin{array}{r}
S=\frac{1}{8} \int_{W^{2}} e^{++} \wedge \Pi \underline{\underline{a}} \tilde{\Sigma}_{\underline{\alpha}}^{\underline{\alpha}} \underline{\underline{\beta}} v_{\underline{\alpha} q}^{-} v_{\underline{\beta} q}^{-}, \\
\Pi \underline{a}=d X^{\underline{a}}-i d \Theta \Sigma \underline{\underline{a}} \theta,
\end{array}
$$

and it is a clear counterpart of (3.15) but in terms of constrained spinors $v_{\underline{\beta}}^{-}$, Eq. (5.1). It can be shown that these spinorial Lorentz harmonics parameterize the celestial sphere $S^{8}$ represented as the Lorentz group coset 51]

$$
\left\{v_{\alpha p}^{-}\right\}=\frac{\operatorname{Spin}(1, D-1)}{[\operatorname{Spin}(1,1) \otimes \operatorname{Spin}(8)] \otimes \mathbb{K}_{8}}=\mathbb{S}^{8},
$$

$\mathbb{K}_{8}$ being an abelian subalgebra. The $D=10$ counterpart of the original pure supertwistor form (2.1) of the supertwistor string action can be obtained by presenting the action (5.2) in the form

$$
S=\int_{W^{2}} e^{++} \wedge\left(d \mu_{q}^{-\underline{\alpha}} v_{\underline{\alpha}}^{-}-\mu_{q}^{-\underline{\alpha}} d v_{\underline{\alpha}}^{-}-i d \chi_{q}^{-} \chi_{q}^{-}\right),
$$

where the $D=10$ counterpart of the Penrose incidence relation reads

$\mu_{q}^{-\underline{\alpha}}=X^{\underline{a}} \tilde{\Sigma}_{\underline{\underline{a}}}^{\underline{\alpha}} \underline{\underline{\beta}} \underline{\underline{\beta}}_{q}^{-}-\frac{i}{2} \Theta^{\underline{\alpha}} \Theta v_{q}^{-}, \quad \chi_{q}^{-}=\Theta^{\underline{\alpha}} v_{\underline{\alpha}}^{-}$.

Due to the basic constraints (5.1), Eq. (5.5) results in $\mu_{q}^{-\underline{\alpha}} v_{\underline{\alpha}}^{-}=X^{\underline{a}} u_{\underline{a}}^{--} \delta_{p q}+\frac{i}{2} \chi_{q}^{-} \chi_{p}^{-}$. This implies that Eq. (5.5), with $v_{\underline{\alpha}} \bar{p}$ constrained by (5.1), provides the general solution of the constraints

$$
\begin{gathered}
\mu_{[q}^{-\underline{\alpha}} v_{\underline{\alpha}}^{-}-\frac{i}{2} \chi_{q}^{-} \chi_{p}^{-}=0, \\
\mu_{(q}^{-\underline{\alpha}} v_{\underline{\alpha} p)}^{-}-\frac{1}{8} \delta_{q p} \mu_{p^{\prime}}^{-\underline{\alpha}} v_{\underline{\alpha} p^{\prime}}^{-}=0,
\end{gathered}
$$


which play the role of the $D=4$ constraint (2.10). More details on the twistor-harmonic formalism in $D=10$ and 11 will be presented in elsewhere.

Let us notice that the necessity of using constrained spinors to describe the higher dimensional generalizations of the twistors was recently noticed 60] in the context of a two-time physics generalization of the Penrose incidence relation, as well as earlier in [42, 48], in relation with the generalization $v_{\underline{\alpha}} \bar{q} v_{\underline{\beta}} \bar{q} \propto p_{\underline{a}} \Sigma_{\underline{\alpha}}^{\underline{\alpha}}, p_{\underline{a}} \delta_{p q} \propto v_{p}^{-} \tilde{\Sigma}_{\underline{a}} v_{q}^{-}$ (cf. (5.1) ) of the $\mathrm{D}=4$ Cartan-Penrose representation of a lightlike momentum, $p_{a} \sigma_{\alpha \dot{\alpha}}^{a}=\lambda_{\alpha} \bar{\lambda}_{\dot{\alpha}}$, the other essential ingredient of the Penrose twistor approach [69]. The twistor transform of the tensionful $D=4, N=1,2 \mathrm{su}-$ perstring actions (4.1) has been presented recently 61 (on the surface of embedding equations $\Pi^{\alpha \dot{\alpha}} v_{\alpha}^{-} \bar{v}_{\dot{\alpha}}^{+}=0$ and $\left.\Pi^{\alpha \dot{\alpha}} v_{\alpha}^{-} \bar{v}_{\dot{\alpha}}^{+}=0\right)$ ).

To conclude, we mention that we did not consider in the present context the interesting problem of the possible stringy origin of the Yang-Mills current part $\int L_{G}$, Eq. (2.12), in the supertwistor string action (2.1). In the light of the discussion in Sec. IV] it is tempting to speculate that (2.12) might originate from the heterotic fermion contribution to the (tensionful) $D=10 \mathrm{~N}=1$ heterotic string action. The main difficulty for such a scenario seems to be the fact that the chirality of the heterotic fermions is opposite to that of the fermionic coordinate function $\Theta^{\underline{\alpha}}$, while the current generating fermions in (2.12) have the same worldsheet chirality [32] as the twistors in (2.1) and the coordinate functions $\theta^{\alpha}, \bar{\theta}^{\dot{\alpha}}$ in (3.1).

\section{Acknowledgements.}

This work has been supported by research grants from the Spanish Ministerio de Educación y Ciencia (FIS200502761 and EU FEDER funds), the Generalitat Valenciana (ACOMP06/187, GV-05/102), the Ukrainian State Fund for Fundamental Research (N 383), and by the EU network MRTN-CT-2004-005104 ('Forces Universe'). One of us (C.M.E.) acknowledges the Spanish M.E.C. for his FPU grant. The authors thank Dima Sorokin for useful comments on the manuscript.
[1] E. Witten, Perturbative gauge theory as a string theory in twistor space, Commun. Math. Phys. 252, 189 (2004) hep-th/0312171.

[2] For a review and earlier references see R. Penrose and M. A. H. MacCallum, Twistor theory: an approach to the quantization of fields and space-time, Phys. Rept. 6, 241 (1972);

R. Penrose, The twistor program, Rep. Math. Phys. 12, 65 (1977).

[3] V. P. Nair, A current algebra for some gauge theory amplitudes, Phys. Lett. B214, 215 (1988).

[4] R. Roiban, M. Spradlin and A. Volovich, A googly amplitude from the B-model in twistor space, JHEP 0404, 012 (2004) hep-th/0402016;

R. Roiban and A. Volovich, All googly amplitudes from the B-model in twistor space, Phys. Rev. Lett. 93, 131602 (2004) hep-th/0402121.

[5] F. Cachazo, P. Svrček and E. Witten, Twistor space structure of one-loop amplitudes in gauge theory, JHEP 0410, 074 (2004) hep-th/0406177.

[6] F. Cachazo, P. Svrček and E. Witten, $M H V$ vertices and tree amplitudes in gauge theory, JHEP 0409, 006 (2004) hep-th/0403047; Gauge theory amplitudes in twistor space and holomorphic anomaly, JHEP 0410, 077 (2004) hep-th/0409245;

F. Cachazo and P. Svrček, Lectures on twistor strings and perturbative Yang-Mills theory, PoS RTN2005, 004 (2005) hep-th/0504194, and refs therein.

[7] I. Bena, Z. Bern, D. A. Kosower and R. Roiban, Loops in twistor space, Phys. Rev. D71, 106010 (2005) hep-th/0410054,

A. Brandhuber, B. Spence and G. Travaglini, From trees to loops and back, JHEP 0601, 142 (2006) hep-th/0510253, and refs. therein.

[8] N. Berkovits and E. Witten, Conformal supergravity in twistor-string theory, JHEP 0408, 009 (2004) hep-th/0406051.

[9] L. J. Mason, Twistor actions for non-self-dual fields: a derivation of twistor-string theory, JHEP 0510, 009 (2005) hep-th/0507269.

[10] G. Chalmers and W. Siegel, The self-dual sector of QCD amplitudes, Phys. Rev. D54, 7628 (1996) hep-th/9606061.

[11] M. Abou-Zeid and C.M. Hull, A chiral perturbation expansion for gravity, JHEP 0602, 057 (2006) hep-th/0511189.

[12] N. Berkovits, An alternative string theory in twistor space for $N=4$ super-Yang-Mills, Phys. Rev. Lett. 93, 011601 (2004) hep-th/0402045.

[13] A. Neitzke and C. Vafa, $N=2$ strings and the twistorial Calabi-Yau, hep-th/0402128

[14] W. Siegel, Untwisting the twistor superstring, hep-th/0404255

[15] O. Lechtenfeld and A. D. Popov, "Supertwistors and cubic string field theory for open $\mathrm{N}=2$ strings," Phys. Lett. B 598, 113 (2004) arXiv:hep-th/0406179.

[16] I. Bars, Twistor superstring in 2T-physics, Phys. Rev. D70, 104022 (2004) hep-th/0407239 Lectures on Twistors, hep-th/0601091.

[17] M. F. Atiyah, N. J. Hitchin, V. G. Drinfel'd and Y. I. Manin, Construction of instantons, Phys. Lett. 65A 185187 (1978)

[18] W. Siegel, Supermulti-instantons in conformal chiral superspace, Phys. Rev. D52, 1042 (1995) hep-th/9412011.

[19] A. Ferber, Supertwistors and conformal supersymmetry, Nucl. Phys. B132, 55 (1978);

T. Shirafuji, Lagrangian mechanics of massless particles with spin, Progr. Theor. Phys. 70, 18 (1983).

[20] A. K. H. Bengtsson, I. Bengtsson, M. Cederwall and N. Linden, Particles, Superparticles And Twistors, Phys. Rev. D36, 1766 (1987);

A. I. Gumenchuk and D. P. Sorokin, Relativistic Dynam- 
ics Of Superparticles And The Twistor Correspondence, Sov. J. Nucl. Phys. 51, 350-355 (1990).

[21] I. A. Bandos and A. A. Zheltukhin, Twistors, harmonics, and zero super-p-branes, JETP Lett. 51, 618 (1990) [Pisma Zh. Eksp. Teor. Fiz. 51, 547 (1990)];

Null super p-brane: Hamiltonian dynamics and quantization, Phys. Lett. B261, 245 (1991);

Covariant quantization of null supermembranes in fourdimensional space-time, Theor. Math. Phys. 88, 925 (1991) [Teor. Mat. Fiz. 88, 358 (1991)].

[22] I.A. Bandos and A.A. Zheltukhin, Null super p-branes quantum theory in four-dimensional space-time, Fortsch. Phys. 41, 619 (1993).

[23] A. P. Isaev and E. A. Ivanov, On sigma model formulation of Green-Schwarz superstring, Mod. Phys. Lett. A4, 351 (1989);

Green-Schwarz superstring as an asymmetric chiral field sigma model, Theor. Math. Phys. 81, 1304 (1990) [Teor. Mat. Fiz. 81, 420 (1989)].

[24] I. A. Bandos and A. A. Zheltukhin, Hamiltonian mechanics and absence of critical dimensions for null membranes, Sov. J. Nucl. Phys. 50, 556 (1989).

[25] D. J. Gross, High-energy symmetries of string theory, Phys. Rev. Lett. 60, 1229 (1988).

[26] U. Lindström and M. Zabzine, Tensionless strings, WZW models at critical level and massless higher spin fields, Phys. Lett. B584, 178 (2004) hep-th/0305098 and refs. therein.

[27] G. Bonelli, On the covariant quantization of tensionless bosonic strings in AdS spacetime, JHEP 0311, 028 (2003) hep-th/0309222.

[28] W. Siegel, Actions for QCD-like strings, Int. J. Mod. Phys. A13, 381 (1998) hep-th/9601002.

[29] K. Bardakci and M. B. Halpern, New dual quark models, Phys. Rev. D3, 2493 (1971).

[30] D. Friedan and P. Windey, Supersymmetric derivation of the Atiyah-Singer index and the chiral anomaly, Nucl. Phys. B235, 395 (1984).

[31] N. Marcus and A. Sagnotti, Group theory from 'quarks' at the ends of strings, Phys. Lett. B188, 58 (1987).

[32] W. Siegel, private communication.

[33] I. A. Bandos, Superparticle in Lorentz harmonic superspace, Sov. J. Nucl. Phys. 51, 906 (1990) ; Multivalued Action Functional, Lorentz Harmonics and Spin, JETP. Lett. 52, 205 (1990) .

[34] A. Bette, J. A. de Azcárraga, J. Lukierski and C. MiquelEspanya, Massive relativistic particle model with spin and electric charge from two-twistor dynamics, Phys. Lett. B595, 491 (2004) arXiv:hep-th/0405166.

[35] I. Bars and M. Picón, Single twistor description of massless, massive, AdS, and other interacting particles, Phys. Rev. D73, 064002 (2006) arXiv:hep-th/0512091.

[36] D. P. Sorokin, V. I. Tkach and D. V. Volkov, Superparticles, Twistors And Siegel Symmetry, Mod. Phys. Lett. A4 (1989) 901.

[37] J. A. de Azcárraga and J. Lukierski, Supersymmetric particles with internal symmetries and central charges, Phys. Lett. B113, 170 (1982); Supersymmetric particles in $N=2$ superspace: phase space variables and hamiltonian dynamics, Phys. Rev. D28, 1337 (1983).

[38] W. Siegel, Hidden local supersymmetry in the supersymmetric particle action, Phys. Lett. 128B, 397-399 (1983); Spacetime-supersymmetric quantum mechanics, Class. Quantum Grav. 2, L95-L97 (1985).
[39] I. Bengtsson and A. A. Zheltukhin, Wess-Zumino actions and Dirichlet boundary conditions for super p-branes with exotic fractions of supersymmetry, Phys. Lett. B570, 222 (2003) hep-th/0306172.

[40] A. A. Zheltukhin and D. V. Uvarov, An inverse Penrose limit and supersymmetry enhancement in the presence of tensor central charges, JHEP 0208, 008 (2002) hep-th/0206214.

[41] A. Sagnotti and M. Tsulaia, On higher spins and the tensionless limit of string theory, Nucl. Phys. B682, 83 (2004) hep-th/0311257.

[42] I. A. Bandos and A. A. Zheltukhin, Spinor Cartan moving $N$ hedron, Lorentz harmonic formulations of superstrings, and kappa symmetry, JETP Lett. 54, 421, (1991); Green-Schwarz superstrings in spinor moving frame formalism, Phys. Lett. B288, 77 (1992).

[43] J.A. de Azcárraga and P.K. Townsend, Superspace geometry and classification of supersymmetric extended objects, Phys. Rev. Lett. 62, 2579-2582 (1989).

[44] I. A. Bandos, J. A. de Azcárraga, M. Picón and O. Varela, Supersymmetric string model with 30 kappa symmetries and extended supersapce and 30-32 BPS states, Phys. Rev. D69, 085007 (2004), hep-th/0307106.

[45] C. Chryssomalakos, J. A. de Azcárraga, J. M. Izquierdo and J. C. Perez Bueno, The geometry of branes and extended superspaces, Nucl. Phys. B567, 293 (2000) hep-th/9904137; J.A. de Azcárraga and J.M. Izquierdo, Superalgebra cohomology, the geometry of extended objects and supermembranes, AIP Conf. Proc. 589, 3-17 (2001) hep-th/0105125.

[46] D. J. Gross, J. A. Harvey, E. J. Martinec and R. Rohm, The heterotic string, Phys. Rev. Lett. 54, 502 (1985).

[47] M. Green, J. Schwarz and E. Witten, Superstring theory, Cambridge Univ. Press, 1999, vol. II p. 269.

[48] I. A. Bandos and A. A. Zheltukhin, D = 10 superstring: Lagrangian and Hamiltonian mechanics in twistor-like Lorentz harmonic formulation, Phys. Part. Nucl. 25, 453 (1994); Lorentz Harmonics and new formulations of superstrings in $D=10$ and supermembranes in $D=11$, Phys. Atom. Nucl. 56, 113 (1993).

[49] A. I. Pashnev and D. P. Sorokin, Note on superfield formulations of $D=2, D=3, D=4, D=6$ and $D=10$ superparticles, Class. Quant. Grav. 10, 625 (1993).

[50] E. Sokatchev, Light cone harmonic superspace and its applications, Phys. Lett. B169, 209 (1986); Harmonic superparticle, Class. Quant. Grav. 4, 237 (1987).

[51] F. Delduc, A. Galperin and E. Sokatchev, Lorentz harmonic (super)fields and (super)particles, Nucl. Phys. B368, 143 (1992);

A. S. Galperin, P. S. Howe and K. S. Stelle, The superparticle and the Lorentz group, Nucl. Phys. B 368, 248 (1992) hep-th/9201020.

[52] C. Fronsdal, Massless particles, orthosymplectic symmetry and another type of Kaluza-Klein theory, in Essays on Supersymmetry (C. Fronsdal ed.), Math. Phys. Stud. 8, D. Reidel Pub. Co. (1986), p. 163.

[53] I. A. Bandos, J. Lukierski and D. P. Sorokin, Superparticle models with tensorial central charges, Phys. Rev. D61, 045002 (2000) hep-th/9904109.

[54] I. Bandos and J. Lukierski, Tensorial central charges and new superparticle models with fundamental spinor coordinates, Mod. Phys. Lett. 14, 1257 (1999) hep-th/9811022.

[55] I.A. Bandos, J.A. de Azcárraga, J.M. Izquierdo and 
J. Lukierski, BPS states in M-theory and twistorial constituents, Phys. Rev. Lett. 86, 4451 (2001) hep-th/0101113.

[56] I. A. Bandos, J. A. de Azcárraga, M. Picón and O. Varela, On BPS preons, generalized holonomies and $D=11$ supergravities, Phys. Rev. D69, 105010 (2004) hep-th/0312266.

[57] I. A. Bandos, BPS preons in supergravity and higher spin theories: An overview from the hill of twistor appraoch, AIP Conf. Proc. 767, 141 (2005) hep-th/0501115.

[58] I. A. Bandos, BPS preons and tensionless super-p-branes in generalized superspace, Phys. Lett. B558, 197 (2003) hep-th/0208110.

[59] J.A. de Azcárraga, J. Gauntlett, J.M. Izquierdo and P.K. Townsend, Topological extensions of the supersymmetry algebra for extended objects, Phys. Rev. Lett. 63, 24432446 (1989)

[60] I. Bars and M. Picón, Twistor transform in d dimensions and a unifying role for twistors, Phys. Rev. D73, 064033 (2006) hep-th/0512348.

[61] D. V. Uvarov, (Super)twistors and (super)strings, hep-th/0601149

[62] Following [1] we use the convention of adding a bar to all the spinors in $\bar{\Upsilon}_{\Sigma}$ rather than associating the bar to the dotted ones (e.g., by denoting $\left(\lambda_{\alpha}, \bar{\mu}^{\dot{\alpha}}\right)$ the two Weyl spinors in $\Upsilon^{\Sigma}$ that make up a Dirac spinor).

[63] The worldsheet local Lorentz $S O(1,1)$ invariance of 2.1 for unconstrained supertwistors holds if $B$ has nontrivial $S O(1,1)$ transformation properties $B \mapsto B+i d \alpha$, so that $B$ is no longer a real connection ( $c f .[12,14])$. Both local symmetries can be maintained if $B$ is taken to be a complex $G L(1, \mathbb{C})$ connection. The reality of the action with complex $B$ would hold if (2.1) were written in the "symmetric' form: $\bar{\Upsilon}_{\Sigma} \nabla \Upsilon^{\Sigma} \mapsto \frac{1}{2}\left(\bar{\Upsilon}_{\Sigma} \nabla \Upsilon^{\Sigma}-\nabla \bar{\Upsilon}_{\Sigma} \Upsilon^{\Sigma}\right)$. For the action (2.1) with a $U(1)$ connection $B$ this holds, up to boundary contributions, provided that $B_{--}^{*}=$ $B_{--}+\frac{i}{2} e_{--}{ }^{m} \nabla_{++} e_{m}^{++}-\frac{i}{2} e_{++}{ }^{m} \nabla_{--} e_{m}^{++}\left(B_{++}\right.$does not enter the action (2.1) ).
[64] In the original paper [12] $\bar{\Upsilon}_{\Sigma}^{-}$, like in (2.14), is the canonically conjugate momentum of $\Upsilon^{-\Sigma}$ but, unlike in (2.14), it is not its complex conjugate; the complex conjugate of $\Upsilon^{-\Sigma}$ is there identified with $\Upsilon^{+\Sigma} \overline{\left(\Upsilon^{-\Sigma}\right.}=\Upsilon^{+\Sigma}$ in [12]). We, however, take the point of view of [14, 16], in which, like in the standard quantization of supertwistors [19], the canonically conjugate supertwistors basically coincide with their complex conjugates.

[65] In the case of particle mechanics, the quantization of $r=2 s$ in units of $\hbar$ can easily be obtained as the requirement that the wave function be well defined as a function of complex variable i.e., that under a $2 \pi$ phase transformation of the bosonic spinor argument the phase of the wave function is shifted by $k r \pi, k \in \mathbb{Z}$. See references and discussion in [33].

For a two-twistor description of massive particles see [34] and references therein; a one-twistor description has recently been developed in [35].

[66] Notice that the open tensionless string in a generalized $D=4, N=1$ superspace enlarged by the tensorial central charge coordinates $y^{m n}=-y^{n m}$ was considered in [39].

[67] The expression of $H_{3}$ shows that it is a ChevalleyEilenberg (CE) three-cocycle for the superspace algebra cohomology [43].

[68] One could also write another twistor-like action for $D=10$ superstrings by using two unconstrained bosonic spinors [49]. In our notation, it reads $\frac{1}{4 \pi \alpha^{\prime}} \int_{W^{2}}\left[e^{++} \wedge \Pi^{a}\left(\lambda^{-} \Sigma_{a} \lambda^{-}\right)-e^{--} \wedge \Pi^{a}\left(\lambda^{+} \Sigma_{a} \lambda^{+}\right)-e^{++} \wedge\right.$ $\left.e^{--}\left(\lambda^{-} \Sigma_{a} \lambda^{-}\right)\left(\lambda^{+} \Sigma^{a} \lambda^{+}\right)\right]-\frac{1}{4 \pi \alpha^{\prime}} \int_{W^{2}} \hat{B}_{2}$. Then, and in contrast with (4.8), the $\kappa$-symmetry of that action would not be irreducible.

[69] Although in $D=6$ and 10 one can use an unconstrained spinor $\lambda_{\alpha}$ to build a light-like vector, $p_{a}=\lambda \Sigma_{a} \lambda \Rightarrow p^{2}=$ 0 , the matrix $p_{a} \Sigma^{a}$ cannot in turn be expressed as $\lambda_{\alpha} \lambda_{\beta}$. 